\title{
Exact Solutions of Discrete Master Equations in Terms of Continued Fractions
}

\author{
G. Haag und P. Hänggi \\ Institut für Theoretische Physik der Universität Stuttgart \\ Stuttgart, Federal Republic of Germany
}

Received March 21, Revised Version May 2, 1979

\begin{abstract}
We present the continued fraction solution for the stationary probability of discrete master equations of one-variable processes. After we elucidate the method for simple birth and death processes we focus the study on processes which introduce at least two-particle jumps. Consequently, these processes do in general not obey a detailed balance condition. The outlined method applies as well to solutions of eigenmodes of the stochastic operator. Further we derive explicit continued fraction solutions for the Laplace transform of conditional probabilities. All the various continued fraction coefficients are given directly in terms of the transition rates and they obey recursion relations. The method is illustrated for the stationary solution of a simple nonlinear chemical reaction scheme originated by Nicolis.
\end{abstract}

\section{Introduction}

In recent years, the concept of master equations has attracted wide attention. These equations play a major role in the description of systems which do not behave in a deterministic way, but display statistical fluctuations of the system variables. In many cases, e.g. if particle or occupation numbers are involved, the fluctuations are of discrete nature. Then a discrete master equation, describing the time evolution of the probability function on a state space $\Sigma$ of a finite or countable set of states, provides a useful starting point for the investigation of the stochastics. Its application covers such different field as quantum optics [1-2], the stochastic theory of chemical reactions [3], biophysical reactions [4] etc. However, exact solutions of master equations are known in relatively simple systems only; mainly because exact solutions in terms of the spectral properties are in general too complicated for an explicit evaluation. As a consequence, various tractable approximative solution schemes [5,6] and the transition factor method [7] have been developed. Nevertheless, it is of great interest to find classes of stochastic processes for which the exact stationary as well as time dependent solutions can be obtained in an analytical form which in addition is also appropriate for a computer evaluation [8]. In this context, the method of continued fractions proved to be a very useful tool for the investigation of correlation functions and spectral functions in stochastic systems [9-11].

In this paper we want to extend the technique of continued fractions to the direct solution of the stationary solution, eigenfunctions and Greensfunctions of discrete master equations: In Sect. 2, we re-examine the steady state solution of a simple birth and death process by deriving explicit continued fraction expansions for the nearest neighbour transition functions. By use of the same reasoning, we present the continued fraction solution of the eigenmodes. By use of the Laplace transform we present in Sect. 3 the explicit continued fraction solutions of the conditional probability. We further investigate the asymptotic behaviour of some stochastic quantities such as the mean absorption time. Sect. 4 contains the main results of the present work. There, we derive the continued fraction solution for the stationary probability and eigenmodes of processes introducing at least two-particle jumps. An example, exposing some advantages of the continued fraction method, is stud- 
ied in Sect. 5. In Sect. 6 we give a brief discussion of the results with an outlook to solutions of more general problems.

\section{Reconsideration of the Steady State Solution of a Simple Birth and Death Process}

In order to elucidate the basic ideas we start the investigation with the stationary solution of a simple birth and death process. Let us consider a process in which a population of size $i, i=0,1, \ldots$ changes due to birth at a rate $r_{i}^{+}=\lambda_{i}$ and death at a rate $r_{i}^{-}=\mu_{i}$ (simple birth and death process). If $P_{i}(t)$ denotes the probability that the population has size $i$ at time $t$ the master equation that governs the evolution of this probability reads

$\dot{P}_{0}(t)=-\lambda_{0} P_{0}(t)+\mu_{1} P_{1}(t)$

$\dot{P}_{i}(t)=\lambda_{i-1} P_{i-1}(t)-\left(\lambda_{i}+\mu_{i}\right) P_{i}(t)+\mu_{i+1} P_{i+1}(t)$,

$i=1,2, \ldots$.

For the following we restrict the consideration to physical systems for which the stationary solution of (2.1) is unique and strictly positive in $\Sigma$. For example, we exclude the case with absorbing states; satisfying $\lambda_{i}=\mu_{i}=0$. Then the nearest neighbour transition function $\xi_{i}^{s}[7]$

$\xi_{i}^{s}=\frac{P_{i}^{s}}{P_{i-1}^{s}}, \quad i=1,2, \ldots$

is well defined. Setting the time-derivation in (2.1) equal to zero we obtain after division with $P_{i-1}^{s}$ the recursion relation

$\xi_{i}^{s}=\frac{\lambda_{i-1}}{\left(\lambda_{i}+\mu_{i}\right)-\mu_{i+1} \xi_{i+1}^{s}}$.

The relation in (2.3) generates the in general infinite continued fraction

$\xi_{i}^{s}=\frac{\lambda_{i-1}}{\left(\lambda_{i}+\mu_{i}\right)-} \frac{\mu_{i+1} \lambda_{i}}{\left(\lambda_{i+1}+\mu_{i+1}\right)-} \ldots, \quad i=1,2, \ldots$

By use of a simple equivalence transformation we can cast (2.4) in the form

$$
\begin{aligned}
& \xi_{i}^{s}=\left(\frac{\lambda_{i-1}}{\mu_{i}}\right) \cdot \frac{1}{1+\frac{\lambda_{i}}{\mu_{i}}-1+\frac{\lambda_{i 1}}{\mu_{i+1}}-} \ldots \\
& \xi_{i}^{s}=\left(\frac{\lambda_{i-1}}{\mu_{i}}\right) \cdot g_{i}^{s} .
\end{aligned}
$$

Observing the boundary condition in (2.1 a) we have

$\xi_{1}^{s}=\frac{\lambda_{0}}{\mu_{1}}$

yielding for the in general infinite continued fraction $g_{1}^{s}$ the result $g_{1}^{s}=1$ and subsequently $g_{i}^{s}=1, i$ $=1,2, \ldots$. For a finite set of $N$ states we have $\lambda_{N}=0$, so that the result $g_{i}^{s}=1$ can be verified explicitly from (2.5). As a consequence we find up to a normalization constant $P_{0}^{s}$ the stationary solution

$P_{n}^{s}=P_{0}^{s} \prod_{i=1}^{n} \xi_{i}^{s}=P_{0}^{s} \exp \sum_{i=1}^{n} \ln \xi_{i}^{s}$

which with (2.6-2.7) combines to

$P_{n}^{s}=P_{0}^{s} \prod_{i=1}^{n} \frac{\lambda_{i-1}}{\mu_{i}}$.

The result in (2.5) is well known from detailed balance considerations.

The same technique can be used for the determination of the components of an eigenmode $\psi_{i}(\rho)$ with eigenvalue $\rho \leqq 0$ obeying:

$0=\lambda_{i-1} \psi_{i-1}(\rho)-\left(\lambda_{i}+\mu_{i}+\rho\right) \psi_{i}(\rho)+\mu_{i+1} \psi_{i+1}(\rho)$.

We remark that with $\lambda_{i}, \mu_{i+1}>0, i=0,1, \ldots$ all eigenvalues $\rho_{i}$ are real and simple. Further the sequence of characteristic polynomials $\varphi_{1}(\rho), \varphi_{2}(\rho), \ldots \varphi_{n}(\rho)$ obtained from the $n$-th approximation where $\lambda_{i}=\mu_{i+1}$ $=0, i=n, \ldots$ forms a so called "Sturm"-chain [12]. We emphasize that the latter properly provides an extremely stable and rapidly convergent algorithm [12] for the calculation of the eigenvalues which occur in (2.10). Introducing

$\xi_{i}^{\rho}=\frac{\psi_{i}(\rho)}{\psi_{i-1}(\rho)}, \quad i=1,2, \ldots$

such that

$\psi_{i}(\rho)=\psi_{0}(\rho) \prod_{j=1}^{i} \xi_{j}^{\rho}$

we immediately find from (2.10) the continued fraction

$\xi_{i}^{\rho}=\frac{\lambda_{i-1}}{\left(\lambda_{i}+\mu_{i}+\rho\right)-} \frac{\mu_{i+1} \lambda_{i}}{\left(\lambda_{i+1}+\mu_{i+1}+\rho\right)-} \ldots$

Noting that for a finite set of $N$ states

$\xi_{1}^{\rho}=\frac{\lambda_{0}+\rho}{\mu_{1}}$

$=\frac{\lambda_{0}}{\left(\lambda_{1}+\mu_{1}+\rho\right)-} \frac{\mu_{2} \lambda_{1}}{\left(\lambda_{2}+\mu_{2}+\rho\right)-} \ldots \frac{\mu_{N-1} \lambda_{N-2}}{\left(\mu_{N-1}+\rho\right)}$. 
(2.13) represents an implicit expression for the characteristic polynomial $\varphi_{N}(\rho)$. The above eigenvector components can also be calculated directly via the recursion formulas for the characteristic polynomials $\varphi_{i}(\rho), i=0,1, \ldots, N$;

$\varphi_{0}(\rho)=1, \quad \varphi_{1}(\rho)=\left(\lambda_{0}+\rho\right)$,

$\varphi_{i}(\rho)=\left(\lambda_{i-1}+\mu_{i-1}+\rho\right) \varphi_{i-1}(\rho)$

$-\lambda_{i-2} \mu_{i-1} \varphi_{i-2}(\rho), \quad i=2, \ldots$

We explicitly find for the components of $\psi^{\rho}$

$\psi_{0}^{\rho}=1$

$\psi_{i}^{\rho}=\varphi_{i}(\rho) /\left(\prod_{j=0}^{i-1} \sqrt{\lambda_{j} \mu_{j+1}}\right) ; \quad i=1,2, \ldots$

However, in contrast to the continued fraction expressions, these explicit formulas (2.14) are known to be numerically unstable [12].

\section{Continued Fractions for Time-Dependent Solutions}

So far we have been concerned with the study of the stationary solution or eigenmodes only. One of the challenges that this work contains is the continued fraction solution of conditional probabilities of a discrete process. Considering the system of differential-difference equations in (2.1) that governs the time-evolution of the probability $P_{n}(t)$ the best approach to a general solution appears to be through the Laplace transform technique. Let us introduce the Laplace transform

$P_{n}(z)=\int_{0}^{\infty} d t e^{-z t} P_{n}(t)$

with $z=x+i y, x>0$. Then the Green's function solution $G\left(n, t \mid n_{0}\right) \equiv P_{n}^{\left(n_{0}\right)}(t)$ (conditional probability) to (2.1) satisfies the set of equations

$\mu_{1} P_{1}^{\left(n_{0}\right)}(z)=-\delta_{0, n_{0}}+\left(\lambda_{0}+z\right) P_{0}^{\left(n_{0}\right)}(z)$,

$\mu_{i+1} P_{i+1}^{\left(n_{0}\right)}(z)$

$=-\lambda_{i-1} P_{i-1}^{\left(n_{0}\right)}(z)+\left(\lambda_{i}+\mu_{i}+z\right) P_{i}^{\left(n_{0}\right)}(z)-\delta_{i, n_{0}}$,

$i=1, \ldots$

In (3.2) $\delta_{i, n_{0}}$ denotes the Kronecker delta function. Choosing the initial condition $\delta_{i, n_{0}}=\delta_{i, n_{0}=0}$ we devise from (3.2a) and (3.2b)

$$
G\left(n=0 \mid n_{0}=0 ; z\right) \equiv P_{0}^{(0)}(z)=\frac{1}{\lambda_{0}+z-\mu_{1} \frac{P_{1}^{(0)}(z)}{P_{0}^{(0)}(z)}}
$$

$$
\begin{aligned}
& =\frac{1}{\lambda_{0}+z-} \frac{\mu_{1} \lambda_{0}}{\lambda_{1}+\mu_{1}+z-} \frac{\mu_{2} \lambda_{1}}{\lambda_{2}+\mu_{2}+z-} \\
& \ldots \frac{\mu_{i} \lambda_{i-1}}{\lambda_{i}+\mu_{i}+z-\mu_{i+1} \frac{P_{i+1}^{(0)}(z)}{P_{i}^{(0)}(z)}} .
\end{aligned}
$$

Thus we obtain for the special solution $P_{0}^{(0)}(z)$ the in general infinite continued fraction

$P_{0}^{(0)}(z)=\frac{1}{\lambda_{0}+z-} \frac{\mu_{1} \lambda_{0}}{\lambda_{1}+\mu_{1}+z-} \ldots$

This continued fraction is just the even contraction of the Stieltjes fraction $[13]^{1}$

$P_{0}^{(0)}(z)=\frac{1}{z+} \frac{\lambda_{0}}{1+} \frac{\mu_{1}}{z+} \frac{\lambda_{1}}{1+} \frac{\mu_{2}}{z+} \ldots$,

which converges uniformly to an analytical function of $z$ in the whole complex phase (except at the poles located on the negative half of the real axis) whenever

$\sum_{i=1}^{\infty}\left(\mu_{1} \mu_{2} \ldots \mu_{i}\right) /\left(\lambda_{0} \lambda_{1} \ldots \lambda_{i}\right)=\sum_{i=1}^{\infty} \frac{M_{i}}{N_{i}}$

$M_{i}=\mu_{1} \mu_{2} \ldots \mu_{i} ; \quad N_{i}=\lambda_{0} \lambda_{1} \ldots \lambda_{i}$

diverges [13]. For the transition function $\xi_{n}^{0}(z)$

$\xi_{n}^{0}(z)=\frac{P_{n}^{(0)}(z)}{P_{n-1}^{(0)}(z)}$

we find from ( $3.2 b)$ the contracted (Stieltjes) continued fraction

$\xi_{n+1}^{0}(z)=\frac{\lambda_{n}}{\lambda_{n+1}+\mu_{n+1}+z-} \frac{\mu_{n+2} \lambda_{n+1}}{\lambda_{n+2}+\mu_{n+2}+z-} \ldots$

For the following, we consider the $n$-th convergent $P_{0, n}^{(0)}(z)$ obtained from (3.6) by setting $\mu_{n}=\mu_{n+1}=\ldots$ $=0$.

In terms of the functions $A_{n}(z), B_{n}(z)$ it can be written as

$P_{0, n}^{(0)}(z)=\frac{A_{n}(z)}{B_{n}(z)}$

where $A_{n}(z), B_{n}(z)$ satisfy the recursion relation [13]

$A_{n+1}(z)=\left(\lambda_{n}+\mu_{n}+z\right) A_{n}(z)-\mu_{n} \lambda_{n-1} A_{n-1}(z)$

$B_{n+1}(z)=\left(\lambda_{n}+\mu_{n}+z\right) B_{n}(z)-\mu_{n} \lambda_{n-1} B_{n-1}(z)$

with

$A_{0}(z)=0, \quad A_{1}(z)=1$

$B_{0}(z)=1, \quad B_{1}(z)=\lambda_{0}+z$.

1 The rates $\lambda_{i}, \mu_{i}$ have been assumed to be positive for all $i$ 
Noticing that

$P_{1}^{(0)}(z)=P_{0}^{(0)}(z) \xi_{1}^{(0)}(z)$

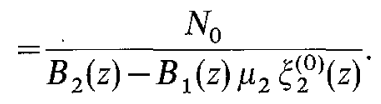

$P_{n}^{(0)}(z)$ is suggested to obey the recursion relation

$P_{n}^{(0)}(z)=\frac{N_{n-1}}{B_{n+1}(z)-B_{n}(z) \mu_{n+1} \xi_{n+1}^{(0)}(z)}$

which is easily verified from (3.2) with induction on $n$. Thus we have for the solution

$P_{n}^{(0)}(z)=P_{0}^{(0)}(z) \prod_{i=1}^{n} \xi_{i}^{(0)}(z)$

the explicit continued fraction

$P_{n}^{(0)}(z)=\frac{N_{n-1} / B_{n}(z)}{B_{n+1}(z) / B_{n}(z)-} \frac{\mu_{n+1} \lambda_{n}}{\lambda_{n+1}+\mu_{n+1}+z-} \ldots$

If we choose in (3.2) the general initial condition $\delta_{n, n_{0}}$ with $n_{0}>0$ induction on $n$ shows that

$B_{n}(z) P_{n-1}^{\left(n_{0}\right)}(z)=\mu_{n} B_{n-1}(z) P_{n}^{\left(n_{0}\right)}(z), \quad n=1,2, \ldots$

In particular for $n=n_{0}$

$B_{n_{0}}(z) P_{n_{0}-1}^{\left(n_{0}\right)}(z)=\mu_{n_{0}} B_{n_{0}-1}(z) P_{n_{0}}^{\left(n_{0}\right)}(z)$.

Inserting (3.18) in (3.2b) with $i=n_{0}$ gives

$\mu_{n_{0}+1} P_{n_{0}+1}^{\left(n_{0}\right)}(z)=\frac{B_{n_{0}+1}(z)}{B_{n_{0}}(z)} \cdot P_{n_{0}}^{\left(n_{0}\right)}(z)-1$.

Equation (3.19) with (3.2b), where $i=n_{0}+1, \ldots$ forms a system of equations analoguous to (3.2b) with $n_{0}$ $=0$. Combining (3.2b) with (3.19) we find the continued fraction

$P_{n_{0}}^{\left(n_{0}\right)}(z)=\frac{1}{B_{n_{0}+1}(z) / B_{n_{0}}(z)-\mu_{n_{0}+1} \xi_{n_{0}+1}^{\left(n_{0}\right)}(z)}$

where $\xi_{n_{0}+1}^{\left(n_{0}\right)}(z)$ is the contracted Stieltjes fraction

$\xi_{n_{0}+1}^{\left(n_{0}\right)}(z)=\frac{\lambda_{n_{0}}}{\lambda_{n_{0}+1}+\mu_{n_{0}+1}+z-} \frac{\mu_{n_{0}+2} \lambda_{n_{0}+1}}{\lambda_{n_{0}+2}+\mu_{n_{0}+2}+z-} \ldots$

By compariance of (3.16) with (3.20-3.21) and applying repeatedly the relation (3.17) we obtain the relations

$P_{i}^{\left(n_{0}\right)}(z)=\frac{M_{n_{0}} B_{i}(z)}{M_{i} B_{n_{0}}(z)} \cdot P_{n_{0}}^{\left(n_{0}\right)}(z), \quad i=0,1, \ldots, n_{0}$

$P_{i}^{\left(n_{0}\right)}(z)=\frac{B_{n_{0}}(z)}{N_{n_{0}-1}} \cdot P_{i}^{(0)}(z), \quad i=n_{0}, n_{0}+1, \ldots$ or

$P_{i}^{\left(n_{0}\right)}(z)$

$=\left\{\begin{array}{l}\frac{M_{n_{0}} B_{i}(z)}{M_{i} B_{n_{0}}(z)}\left(\frac{1}{B_{n_{0}+1}(z) / B_{n_{0}}(z)-} \frac{\mu_{n_{0}+1} \lambda_{n_{0}}}{\left.\left(\lambda_{n_{0}+1}+\mu_{n_{0}+1}+z\right)-\ldots\right)}\right. \\ i=0,1, \ldots, n_{0} \\ \frac{N_{i-1} B_{n_{0}}(z)}{N_{n_{0}-1} B_{i}(z)}\left(\frac{1}{B_{i+1}(z) / B_{i}(z)-} \frac{\mu_{i+1} \lambda_{i}}{\left(\lambda_{i+1}+\mu_{i+1}+z\right)-} \ldots\right), \\ i=n_{0}, n_{0}+1, \ldots\end{array}\right.$

In time space we have for the time-homogeneous conditional probability $G\left(n, t \mid n_{0}\right)$ from the inverse Laplace transformation

$G\left(n, t \mid n_{0}\right)=\frac{1}{2 \pi} \int_{-\infty}^{+\infty} e^{z t} P_{n}^{\left(n_{0}\right)}(z) d y$.

In order to calculate this Laplace inversion one advantageously determines first the location of the poles (eigenvalues $\rho \leqq 0$ ). Using the $m$-th convergent approximation $P_{n, m}^{\left(n_{0}\right)}(z)$ the eigenvalues are according to (3.16) given by the zeros of the polynomials $B_{n+m}(z)$.

It is also worthwhile to emphasize that the continued fraction solutions allow an efficient calculation of asymptotic stochastic properties. For example, we find from the residue of $P_{i}^{\left(n_{0}\right)}(z)$ the correctly normalized stationary probability

$\lim _{z \rightarrow 0} z P_{i}^{\left(n_{0}\right)}(z)=P_{i}^{s}$

and from

$-\lim _{z \rightarrow 0} \frac{\partial}{\partial z} z P_{i}^{\left(n_{0}\right)}(z)=\tau_{n_{0}}(i)$

the mean absorption time $\tau_{n_{0}}(i)$

$\tau_{n_{0}}(i)=\int_{0}^{\infty} t \frac{\partial}{\partial t} G\left(i t \mid n_{0}\right) d t$

\section{Stationary Solution of a Discrete Master Equation with Two-Particle Jumps}

In this section we investigate the stationary stochastics of systems for which detailed balance does not necessarily hold. In particular, we confine ourselves to a study of systems in which two-particle jumps with rates $r_{i}^{++}=v_{i}$ and $r_{i}^{--}=\omega_{i}$ may occur. Examples are given by the stochastic modelings of interacting social groups [14-15] or chemical reactions introducing bimolecular reaction steps $[3,5,16]$. For these cases the stationary birth and death master equation 
reads

$0=-[0] P_{0}^{s}+\mu_{1} P_{1}^{s}+\omega_{2} P_{2}^{s}$

$0=\lambda_{0} P_{0}^{s}-[1] P_{1}^{s}+\mu_{2} P_{2}^{s}+\omega_{3} P_{3}^{s}$

$0=v_{i-2} P_{i-2}^{s}+\lambda_{i-1} P_{i-1}^{s}-[i] P_{i}^{s}$

$+\mu_{i+1} P_{i+1}^{s}+\omega_{i+2} P_{i+2}^{s}, \quad i=2,3, \ldots$

with

$[0]=\lambda_{0}+v_{0}$

$[1]=\lambda_{1}+\mu_{1}+v_{1}$

$[i]=\lambda_{i}+\mu_{i}+v_{i}+\omega_{i}, \quad i=2,3, \ldots$.

As before, we introduce the nearest-neighbour transition function $\xi_{i}^{s}=P_{i}^{s} / P_{i-1}^{s}$. In virtue of (4.1 a) we obtain

$\xi_{1}^{s}=\frac{[0]}{\mu_{1}+\omega_{2} \xi_{2}^{s}}$

and from the boundary condition in (4.1b) we deduce

$\xi_{2}^{s}=\frac{[1][0]-\mu_{1} \lambda_{0}}{\omega_{2} \lambda_{0}+[0]\left(\mu_{2}+\omega_{3} \xi_{3}^{s}\right)}$.

Considering the expression for $\xi_{3}^{s}$ we find after a straightforward calculation the result

$$
\begin{aligned}
& \xi_{3}^{s}=\frac{[2][1][0]-[2] \mu_{1} \lambda_{0}-[1] v_{0} \omega_{2}-}{\omega_{3}\left(\lambda_{1}[0]+\mu_{1} v_{0}\right)+} \\
& \frac{-[0] \mu_{2} \lambda_{1}-\lambda_{0} \lambda_{1} \omega_{2}-v_{0} \mu_{1} \mu_{2}}{+\left([1][0]-\mu_{1} \lambda_{0}\right)\left(\mu_{3}+\omega_{4} \xi_{4}^{s}\right)} .
\end{aligned}
$$

This result suggests the following ansatz for the recursion relation of the transition coefficient $\xi_{n}^{\mathrm{s}}$

$\xi_{n}^{s}=\frac{a_{n-1}}{b_{n}+a_{n-2}\left(\mu_{n}+\omega_{n+1} \xi_{n+1}^{s}\right)}$

with

$a_{-1}=1, \quad a_{0}=[0], \quad a_{1}=[1][0]-\mu_{1} \lambda_{0}$,

$b_{1}=0, \quad b_{2}=\omega_{2} \lambda_{0}$.

By use of (4.1c) and induction on $n$ we find the recursion relation

$a_{n-1}=[n-1] a_{n-2}-\omega_{n-1} v_{n-3} \frac{a_{n-2} a_{n-4}}{a_{n-3}}$
$-\left(b_{n-1}+\mu_{n-1} a_{n-3}\right) \cdot\left(v_{n-3} \frac{b_{n-2}+\mu_{n-2} a_{n-4}}{a_{n-3}}+\lambda_{n-2}\right)$,

$b_{n}=\omega_{n}\left(v_{n-3}\left[b_{n-2}+\mu_{n-2} a_{n-4}\right]+\lambda_{n-2} a_{n-3}\right)$.
With an equivalence transformation we can cast (4.6) in the form

$\xi_{n}^{s}=\alpha_{n} \frac{1}{1+} \frac{\beta_{n}}{1+} \frac{\beta_{n+1}}{1+} \ldots$

where

$\alpha_{n}=\frac{a_{n-1}}{b_{n}+\mu_{n} a_{n-2}} ;$

$\beta_{n}=\omega_{n+1} \frac{a_{n} a_{n-2}}{\left(b_{n}+\mu_{n} a_{n-2}\right)\left(b_{n+1}+\mu_{n+1} a_{n-1}\right)}$.

Considering a (right-) eigenmode $\psi(\rho)$ we obtain for the ratio $\xi_{n}(\rho)$ the continued fraction

$\xi_{n}(\rho)=\frac{\psi_{n}(\rho)}{\psi_{n-1}(\rho)}=\frac{a_{n-1}(\rho)}{b_{n}(\rho)+a_{n-2}(\rho)\left(\mu_{n}+\omega_{n+1} \xi_{n+1}(\rho)\right)}$

where $a_{n}(\rho), b_{n}(\rho)$ satisfy the recursion relations in (4.8) with $[n]$ substituted by $[n] \rightarrow[n]+\rho$.

In analogy to Sect. 3, where we find from (3.14), (3.24) a closed continued fraction for the stationary solution itself, we may look for a closed expression for the stationary solution of the process in (4.1). The results in (4.3-4.5) suggest the following ansatz which is verified by induction on $n$.

$P_{n}^{s}=P_{0}^{s} \frac{c_{n-1}}{d_{n}+d_{n-1} a_{n-2} \omega_{n+1} \xi_{n+1}}$.

Here the continued fraction coefficients $\left\{c_{n}\right\},\left\{d_{n}\right\}$ obey the relations

$c_{n}=a_{0} \cdot a_{1} \ldots a_{n}, \quad n=0,1,2, \ldots$

$d_{n}=\left(b_{n}+a_{n-2} \mu_{n}\right) d_{n-1}+\omega_{n} a_{n-1} a_{n-3} d_{n-2}$

with the initial values

$d_{0}=1, \quad d_{1}=\mu_{1}$.

In the particular case that $\omega_{i}=0, i=2,3, \ldots N-1,{ }^{2}$ we obtain from (4.12 4.14) for the distribution function $P_{n}^{s}$ the useful result

$P_{n}^{s}=P_{0}^{s} \frac{a_{n-1}}{\mu_{1} \cdot \mu_{2} \cdot \ldots \cdot \mu_{n}}, \quad n=1,2, \ldots$.

For a simple birth and death process we immediately recover the well known result in (2.9).

\section{Application to a Simple Nonlinear Model}

We illustrate here some of the previous ideas on the simple biomolecular reaction scheme

$A \stackrel{k_{1}}{\longrightarrow} X, \quad 2 X \stackrel{k_{2}}{\longrightarrow} A$.

${ }^{2}$ We assume a finite number of states. Then no explosion to infinity can occur 
This reaction has been studied previously by a number of authors $[5,16,17]$. For the following we denote by $n$ the number of particles of a chemical species $X$ which is the stochastic variable of the model. The master equation for this model reads

$\dot{P}(n, t)=k_{1} N_{A} P(n-1, t)+k_{2}(n+1)(n+2) P(n+2, t)$

$-\left[k_{1} N_{A}+k_{2}(n-1) n\right] P(n, t)$

yielding

$\lambda_{n}=k_{1} N_{A} \equiv \lambda, \quad \mu_{n}=0$,

$v_{n}=0, \quad \omega_{n}=k_{2} n(n-1), \quad n=2,3, \ldots$.

The continued fraction coefficients $\left\{a_{n}, b_{n}\right\}$ in (4.8) are calculated to be

$a_{n}=\lambda^{n+1}, \quad b_{n}=\omega_{n} \lambda^{n-1}$

such that from $(4.10)$

$\alpha_{n}=\beta_{n}=\frac{\left(\lambda / k_{2}\right)}{n(n-1)}, \quad n=2,3, \ldots$

Thus Equation (4.6) reads explicitly

$\xi_{n}^{s}=\frac{\left(\lambda / k_{2}\right)}{n(n-1)+(n+1) n \xi_{n+1}^{s}}, \quad n=1,2, \ldots$

The maximum of the probability distribution can be obtained from $(5.6)$ by setting $\xi_{n}^{s} \approx \xi_{n+1}^{s} \approx 1$. The position of the peak in the example is thus given by

$n_{w}=\left(\frac{\lambda}{2 k_{2}}\right)^{\frac{1}{2}}$.

In normal situations the peak has a rather broad extension on the macroscopic scale of the particle number $n$. Because $\xi_{n}^{s}$ around $n_{w}$ is a slowly varying quantity we set in roughest approximation

$\xi_{n_{w} \pm \Delta n}^{s} \approx \frac{\left(\lambda / k_{2}\right)}{2(n \pm \Delta n)^{2}}$.

A more refined expression for $\xi_{n}^{s}$ can be obtained from (5.6) with $\xi_{n}^{s} \approx \xi_{n+1}^{s}$

$\xi_{n}^{s}=-\frac{1}{2}+\left(\frac{1}{4}+\frac{\lambda}{k_{2}} \frac{n^{2}+1}{\left(n^{2}-1\right)^{2}}\right)^{\frac{1}{2}} \approx-\frac{1}{2}+\left(\frac{1}{4}+\frac{\lambda}{k_{2}} \frac{1}{n^{2}}\right)^{\frac{1}{2}}$.

This expression just coincides with the result obtained by Görtz and Walls in terms of the $q$-method [5]. But in contrast to the $q$-method we do not need to consider a quartic equation in $q$.

Noting that with the Euler-Mac Laurin summation formula we obtain

$$
P_{n_{w}+\Delta n}^{s}=\left.P_{n_{w}}^{s} \prod_{i=n_{w}+1}^{n_{w}+\Delta n} \xi_{i}^{s} \approx P_{n_{w}}^{s} \exp \frac{1}{2} \frac{\partial \xi_{n}^{s}}{\partial n}\right|_{n=n_{w}} \cdot(\Delta n)^{2} .
$$

Therefore we have for the variance $\sigma^{2}$ of the distribution from (5.9) the result

$\sigma^{2}=\left\langle(\Delta n)^{2}\right\rangle=\frac{3}{4}\left(\frac{\lambda}{2 k_{2}}\right)^{\frac{2}{2}}=\frac{3}{4} n_{w}$.

Using for large and small $n$ the somewhat crude approximation $\xi_{n}^{s}$ in (5.8) for all $n^{3}$

$\xi_{n}^{s}=\frac{\left(\lambda / k_{2}\right)}{2 n^{2}}, \quad n=1,2, \ldots$

we obtain for the stationary probability the expression

$P_{n}^{s}=P_{0}^{s}\left(\frac{\lambda}{2 k_{2}}\right)^{n} \cdot \frac{1}{(n !)^{2}}$.

By noting the ascending series of the modified first Bessel function [18] we find for $P_{0}^{s}$ the result

$P_{0}^{s}=\frac{1}{I_{0}\left(\sqrt{2 \lambda / k_{2}}\right)}$.

Henceforth, the mean values $\langle n\rangle$ and $\left\langle n^{2}\right\rangle$ are calculated to be

$\langle n\rangle=n_{w} \frac{I_{1}\left(2 n_{w}\right)}{I_{0}\left(2 n_{w}\right)}=n_{w}-\frac{1}{4}+\ldots$,

$\left\langle n^{2}\right\rangle=n_{w}^{2}$

Thus the variance $\sigma^{2}$ equals within this crude approximation the value

$\sigma^{2}=\left\langle n^{2}\right\rangle-\langle n\rangle^{2}=\frac{1}{2} n_{w}+\ldots$

For the model in (5.1) the moments can be calculated exactly [17] yielding

$\langle n\rangle=n_{w} \frac{I_{0}\left(4 n_{w}\right)}{I_{1}\left(4 n_{w}\right)}=n_{w}+\frac{1}{8}$,

$\sigma^{2}=n_{w} \frac{I_{0}\left(4 n_{w}\right)}{I_{1}\left(4 n_{w}\right)}\left(1-n_{w} \frac{I_{0}\left(4 n_{w}\right)}{I_{1}\left(4 n_{w}\right)}\right)$

$+n_{w}^{2}=\frac{3}{4} n_{w}+\frac{1}{16}+\ldots$.

Thus, we can deduce, that with a reasonable size for the system (e.g. $\langle n\rangle \approx 100)$ the Gaussian approximation (5.10-5.11) is almost exact! With the crude approximation in (5.12) the mean value is produced nearly exact whereas the variance fails the exact result by a factor $\frac{1}{4} n_{w}$.

3 For $n \gg 1$ the correct asymptotic behaviour is from (4.6) given by $\xi_{n}=\lambda /\left(k_{2} n^{2}\right)$ 


\section{Discussion}

We derived exact solutions in terms of continued fractions of discrete one-variable master equations which in general do not satisfy a detailed balance relation.

The explicit expressions are given in mathematical appealing forms which simplify considerably the study of the stationary and time-dependent fluctuation dynamics in nonlinear stochastic systems. This latter fact is also exposed in the example of Sect. 5 , where we obtain very satisfactory results by use of simple approximation schemes. The results of a nontrivial application and various refined approximation schemes will be discussed elsewhere.

The outlined method for the stationary solution is not restricted to birth and death processes with possible two-particle jumps only. However, higher order jump processes require a study of more and more complicated boundary conditions. Likewise, the method in Sect. 3 can also be extended to higher order jump processes. For example, we obtain from the results in Sect. 4 for the transition function of the conditional probability of the two-particle jump process the recursion relation

$$
\xi_{i}^{\left(n_{0}\right)}(z)=\frac{P_{i}^{\left(n_{0}\right)}(z)}{P_{i-1}^{\left(n_{0}\right)}(z)}=\frac{a_{i-1}(z)}{b_{i}(z)+a_{i-2}(z)\left(\mu_{i}+\omega_{i+1} \xi_{i+1}^{\left(n_{0}\right)}(z)\right)},
$$

$i=1, \ldots, n_{0}-1, n_{0}+1, \ldots$

Hereby $a_{i}(z), b_{i}(z)$ satisfy the recursion relation in (4.8) with $[i]$ substituted by $[i(z)]=[i]+z$. In this context it is worthwhile emphasizing that for the calculation of correlation functions $\langle f(x(T)) g(x(0))\rangle$ the direct continued fraction methods in [9-11] are in general superior to those which introduce the continued fraction representation of the conditional probability: Whereas the correlation function may be determined by a restricted set of relaxation frequencies and variable weighted modes, the conditional probability is composed of all relaxation frequencies and modes with equal probability.

\section{References}

1. Haken, H.: Encyclopedia of Physics, Vol XXVI2c. Berlin, Heidelberg, New York: Springer 1970

2. Agarwal, G.S.: Springer Tracts in Modern Physics 70, Berlin, Heidelberg, New York: Springer 1974

3. Mc Quarrie, D.A.: J. Appl. Prob. 4, 413 (1967)

4. Goel, N.W., Richter-Dyn, N.: Stochastic Models in Biology New York: Academic Press 1974

5. Görtz, R., Walls, D.F.: Z. Physik B25, 423 (1976)

6. Haag, G., Weidlich, W., Alber, P.: Z. Physik B26, 207 (1977)

7. Haag, G.: Z. Physik B29, 153 (1978)

8. Weidlich, W.: Z. Physik B30, 345 (1978)

9. Hänggi, P., Rösel, F., Trautmann, D.: Z. Naturforsch. 33a, 402 (1978)

10. Grossmann, S., Schranner, R.: Z. Physik B30, 325 (1978)

11. Hänggi, P.: Z. Naturforsch. 33a, 1380 (1978)

12. Schwarz, H.R., Rutishauser, H., Stiefel, E.: Matrizen-Numerik p. 135, Stuttgart: B.G. Teubner 1968

13. Perron, O.: Die Lehre von den Kettenbrüchen: Leipzig-Stuttgart: B.G. Teubner 1913

14. Weidlich, W.: Collect. Phenom. 1, 51 (1972)

15. Walls, D.F.: Collect. Phenom. 2, 125 (1976)

16. Nicolis, G.: J. Statist. Phys. 6, 195 (1972)

17. Mazo, R.M.: J. Chem. Phys. 62, 4244 (1975)

18. Abramowitz, M., Stegun, I.A.: Handbook of Mathematical Functions. U.S. Department of Commerce, N.B.S. Appl. Math. 55, 374 (1964)

\author{
G. Haag \\ P. Hänggi \\ Institut für Theoretische Physik \\ Universität Stuttgart \\ Pfaffenwaldring 57 \\ D-7000 Stuttgart 80 \\ Federal Republic of Germany
}

